\title{
Du message tambouriné au message électronique
}

Changements engendrés ces dernières années par les nouveaux outils de communication dans la région centre du Sepik (Papouasie NouvelleGuinée)

\section{Christian Coiffier}

\section{(2) OpenEdition}

\section{Journals}

Édition électronique

URL : http://journals.openedition.org/jso/8787

DOI : $10.4000 /$ jso.8787

ISSN : 1760-7256

Éditeur

Société des océanistes

Édition imprimée

Date de publication : 15 juillet 2018

Pagination : 245-258

ISBN : 978-2-85430-135-9

ISSN : 0300-953x

Référence électronique

Christian Coiffier, « Du message tambouriné au message électronique », Journal de la Société des Océanistes [En ligne], 146 | 2018, mis en ligne le 15 juillet 2020, consulté le 23 juillet 2020. URL : http:// journals.openedition.org/jso/8787 ; DOI : https://doi.org/10.4000/jso.8787

(C) Tous droits réservés 


\title{
Du message tambouriné au message électronique. Nouveaux outils de communication dans le Centre Sepik (PNG)
}

par

\author{
Christian COIFFIER
}

\begin{abstract}
In about a decade mobile phones and Internet have considerably changed the everyday life of the people living in the villages of the Sepik river valley. These new technologies follow many others, which profoundly modified the structures of local societies in almost a century. This study focuses mainly on the Iatmul village of Palimbei and its neighbourhood in the East Sepik Province. Having presented the former technique of communication between villages by drummed message. This study recount the history of how new electronic media (television, computer, Internet...) have been implemented in the region, with particular attention to mobile telephony. This study analyze the advantages and disadvantages for the inhabitants in a context of important environmental changes, such as deforestation and mining projects.
\end{abstract}

KEYwORDS: mobile phone, Internet, computer, new technologies, socio-economic development, Sepik Province, Iatmul people

En un siècle, les peuples de la vallée du Sepik en Papouasie Nouvelle-Guinée se sont adaptés très rapidement aux nouvelles techniques venues d'autres continents, qui ont considérablement changé leurs modes de vie quotidiens. Citons, entre autres, l'utilisation d'outils en métal, l'éclairage à l'aide de lampes à pétrole (Coiffier, 1999: 88-89), le moteur à essence pour la propulsion des pirogues (Coiffier, 1995: 69-75), la consommation de nourritures en conserve (Coiffier, 1998 : 352-353). Les nouvelles techniques de l'infor-

\section{RÉSUMÉ}

Depuis une dizaine d'années le téléphone mobile et internet sont venus bouleverser la vie quotidienne des peuples vivants dans les villages de la vallée du fleuve Sepik. L'apparition de ces nouvelles technologies fait suite à beaucoup d'autres qui, en près d'un siècle, ont profondément modifié les structures des sociétés locales. Cette étude se focalise principalement sur le village iatmul de Palimbei et ses environs dans la province du Sepik de l'Est. Après avoir présenté l'ancienne technique de communication entre villages par message tambourinés elle retrace l'historique de l'implantation dans cette région de nouveaux matériels électroniques (télévision, ordinateur, Internet...) et tout particulièrement de la téléphonie mobile. Cette étude en analyse les avantages et les inconvénients pour les habitants dans un contexte de grands changements environnementaux comme la déforestation et les projets miniers.

Mots-CLÉs : téléphone mobile, internet, ordinateur, nouvelles technologies, développement socioéconomique, province du Sepik, Iatmul

mation et de la communication (téléphonie mobile et internet) leurs permettent maintenant d'être en relation avec le reste du monde. Depuis la fin de la guerre du Pacifique en 1946, certains jeunes de cette région, scolarisés dans les écoles missionnaires puis dans les universités pour certains d'entre eux, sont partis à la découverte d'autres provinces de leur pays, de l'Australie ou d'autres continents. Ils ont ainsi pu transmettre aux membres de leur famille un nouveau regard sur le monde. Dès les années 1960, Edmund Carpenter ${ }^{1}$ fut

1. Edmund Carpenter et son épouse, la photographe Adélaide de Menil, réalisèrent des recherches sur le terrain dans diverses régions du Sepik en 1969.

* Attaché au MNHN (Éco-Anthropologie et Ethnobiologie 72 06), coiffier@mnhn.fr 
l'un des premiers à s'intéresser aux effets des médias sur les populations du Sepik (Mc Luhan, 1964).

La fréquentation régulière depuis 1972 et durant plus de quarante années d'une petite communauté iatmul de plusieurs centaines de personnes vivant dans le village de Palimbeï et son hameau satellite au bord du fleuve Sepik, Abusatngei ${ }^{2}$, m'a permis d'observer au fil du temps les différents effets de l'importation des nouvelles technologies. Cet article examine le développement de la téléphonie mobile et d'internet en se limitant à cette seule région du Moyen-Sepik, tout en présentant quelques éléments comparatifs avec des recherches effectuées par des collègues dans d'autres régions de Nouvelle-Guinée, des Salomon et du Vanuatu.

\section{Implantation des technologies de l'information et de la communication dans la région}

Depuis plusieurs siècles, les habitants de la côte nord de Nouvelle-Guinée utilisent les tambours à fente (appelés garamut en pidgin-english) pour communiquer à distance grâce à un système de signaux tambourinés. Ces derniers ont été étudiés par Thomas Aitken (1980) ainsi que par Hugo Zemp et Christian Kaufmann (1969). Ces grands tambours à fente de deux à quatre mètres de longueur, taillés dans une section d'arbre kwarap (Intsia bijuga), au bois extrêmement dur et imputrescible, permettent encore de nos jours aux villageois de rythmer les danses cérémonielles et d'envoyer des messages codés $(\text { tavit })^{3}$ à d'autres villages éloignés de plusieurs kilomètres à vol d'oiseau. Les sons de ces tambours sont tous différents si bien que les villageois en distinguent aisément la provenance comme jadis les habitants de nos villages européens distinguaient les divers tintements des cloches de leurs églises respectives. Ces tambours sont conservés dans les maisons des hommes (Coiffier, 1994 : 857) et chaque clan en possède théoriquement une paire. Ils sont utilisés horizontalement avec un ou deux exécutants munis d'un ou de deux maillets chacun pour frapper sur la surface extérieure ou carrément à l'intérieur de la partie creuse. En général ces tambours ont une importance extrême car ils sont considérés comme des esprits wagan (Bateson, 1971 : 236). Les sons provoqués par la frappe des maillets sont alors la voix de ces esprits. Les sculptures qui ornent ces tambours et la forme phallique des maillets, leurs donnent une connotation sexuelle (Coiffier, 1994 : 873). Les chefs de famille utilisent encore parfois des tambours plus petits d'environ un mètre de longueur destinés uniquement à la transmission de messages personnels et chaque homme possède son tempo identitaire. C'est ainsi que, jusqu'à la fin du siècle dernier, les habitants de la vallée du Sepik communiquaient d'un village à l'autre ou de personne à personne. La comparaison entre cette technique et la téléphonie mobile qui a été faite par certains (Watson and Duffield, 2015) est contestée par Borut Telban et Daniela Vavrova (2014 : 226-227) qui pensent qu'imaginer les téléphones mobiles d'aujourd'hui comme des objets modernes remplaçant les tambours à fente est une erreur. Pour ces chercheurs, ces deux instruments ont des fonctions très différentes. Les téléphones mobiles sont en effet des objets personnels manufacturés à l'étranger alors que les tambours à fente sont fabriqués localement selon des règles strictes et transmettent des messages compréhensibles par les seuls initiés. Le terme attribué au téléphone mobile par les lettrés iatmul est wolinemba smi, que l'on peut traduire par "le tambour des Blancs "; mais le nom usuel utilisé par tous en pidgin-english est mobael. Pour les scientifiques les messages tambourinés sont transmis par des ondes sonores alors que les messages vocaux ou électroniques sont transmis par des ondes électromagnétiques. Pour de nombreux villageois du Sepik les tambours $g a-$ ramut sont encore maintenant en relation avec les esprits ancestraux et demeurent ainsi la propriété des divers clans. Constatons donc que le tambour à fente comme le téléphone mobile sont deux instruments de communication à distance qui entretiennent quelques liens de filiation au vu de leur utilisation pour rentrer en contact avec l'au-delà, au sens littéral du terme!

Il y quarante ans, la majorité des échanges de produits alimentaires se faisait avec les communautés voisines alors que les relations économiques avec les peuples de la côte nécessitaient des échanges en chaîne avec diverses communautés intermédiaires. De nos jours, les déplacements entre les divers villages du fleuve s'effectuent encore en pirogue et à pied. À partir des années 1960, les pirogues ont commencé à être motorisées et les déplacements lointains sont devenus plus aisés grâce à la construction de routes entre la capitale provinciale Wewak, et divers sites au bord du fleuve Sepik ; Pagwi-Burui, Timbunke et Angoram, ce qui a participé à désenclaver la région. Les nouvelles locales se transmettaient cependant de personne à personne au gré de leurs déplacements; ce que les Français des îles appellent, Radio-cocotier. C'étaient souvent les membres des missions religieuses qui assuraient, et qui assurent encore aujourd'hui, la déserte des

2. Mes remerciements vont à la famille de Benny Kusodimi et son épouse Martha Mbaomilagwa qui m’accueillent dans leur maison depuis 1980 et qui m’ont adopté en 1987. Cette dernière enquête a été réalisée au mois d'août 2015 dans le village d'Abusatngei et ses environs. Ma gratitude s’adresse également à Patrick Boursin, Jean Coiffier, Thierry Morant et Vivian Saminaden qui ont aimablement accepté de relire ce texte, de faire des corrections ainsi que la traduction en langue anglaise du résumé.

3. Tous les termes vernaculaires cités dans cet article appartiennent à la langue Iatmul centrale (Palimbeï kwinti). 


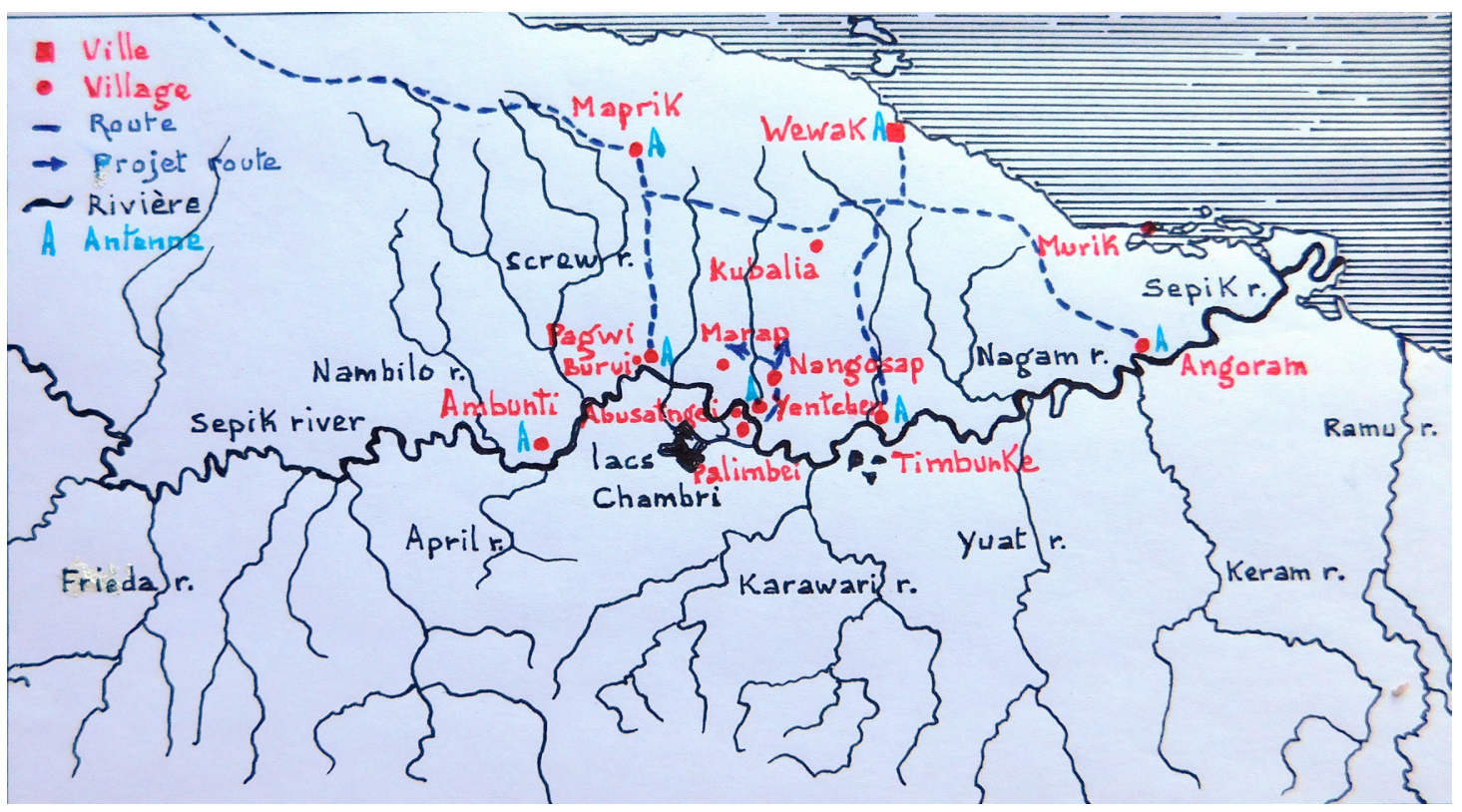

Carte 1. - Schéma de la région montrant les routes et les lieux d'implantation des antennes-relais évoquées dans ce texte (C) C. Coiffier, 2017)

courriers (lettres et petits paquets) aux habitants des villages du bord du fleuve.

Depuis une cinquantaine d'années, l'influence des technologies de l'information et de la communication sur le développement de la région n’a cessé de croître (Logan, 2012). Jusqu’au début du $\mathrm{XXI}^{\mathrm{e}}$ siècle les échanges de communications verbales pouvaient passer par les ondes radio, mais seules les missions installées à Burui, Chambri ou Timbunke et quelques rares marchands australiens ou locaux possédaient des postes émetteurs-récepteurs. Les villageois avaient connaissance de l'existence des talkies walkies, qu'ils voyaient utilisés par les marins des navires de touristes, comme le $\mathrm{Me}$ lanesian Explorer, mais aucun d'entre eux n'avait acquis ce type d'appareil à notre connaissance. Dans les années 1970-80 de nombreux hommes ont acheté des postes de radio portables fonctionnant avec des batteries, ce qui leur a permis d'écouter de la musique locale ou étrangère. Ces voix venant de loin étaient appelées wali kuasa (la chose de là-haut qui vient des Blancs) ou waare mendje ${ }^{4}$ (la cordelette de sons). La possession d'une radio portable pour les jeunes hommes était un atout de poids pour conquérir les faveurs d'une femme désirée. Les villageois du Sepik ont pu ainsi accéder aux informations, tant nationales que locales, particulièrement avec l'émission provinciale très populaire « Tok Save » qui diffusait des messages de particulier à particulier.

C'est au début du XXI ${ }^{\mathrm{e}}$ siècle au sein du bidonville de Bumbu à Wewak dans la maison de Saoun ${ }^{5}$ originaire du village de Palimbeï que j'ai vu le premier téléviseur accompagné d'un lecteur-enregistreur de cassettes vidéo. Les jeunes se sont ainsi très vite habitués à regarder les films hollywoodiens, les informations et les publicités sur la chaîne nationale ou la chaîne australienne. Le premier téléviseur avec lecteur-enregistreur de cassettes vidéo à Abusatngei fut acheté par Philippe Aveyumbum (frère aîné de Benny Kusodemi) en 1999 à l'occasion d'un séjour à Djayapura en West Papua ${ }^{6}$. Une petite case au toit surmonté d'une antenne parabolique fut construite pour y placer le téléviseur, le lecteur-enregistreur et le groupe électrogène pour les alimenter. Moyennant une somme modique les habitants $\mathrm{du}$ hameau et particulièrement les plus jeunes venaient assister à la retransmission de matchs de football. La majorité des nouveaux objets comme les caméra, téléviseur ou ordinateur sont dénommés en anglais ou en pidgin-english et ils n'ont pas de traduction en langue vernaculaire, vraisemblablement car ils n'ont pas d'équivalents locaux et ne sont que très peu utilisés dans les villages (Coiffier, 1999: 90-94).

4. Les cordelettes mendje sont constituées de deux torons de fibres végétales et servent à la confection des filets. Elles constituent ainsi une image locale représentative des ondes sonores.

5. Saoun, cousin germain de Mbaomilagwa, a marié une de ses filles à un entrepreneur chinois de Singapour dont elle a eu plusieurs enfants. Le père qui ne vit plus à Wewak envoie régulièrement de l'argent pour l'éducation des enfants et c'est lui qui a offert le téléviseur.

6. La frontière routière étant fermée à cette époque c'est en bateau que s'effectuait tout un trafic entre Djayapura capitale de la province indonésienne de West Papua, et Aïtape, port de Papouasie Nouvelle-Guinée, situé non loin de cette frontière. 


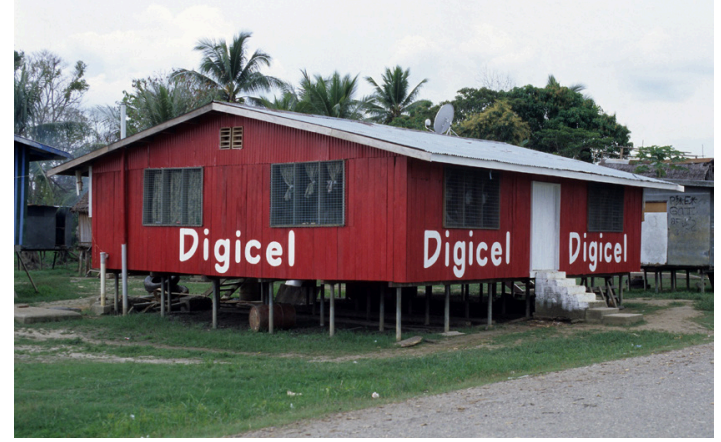

Рното 1. - Publicité de l'opérateur Digicel sur une maison au village de Pagwi (C C. Coiffier, 2010)

\section{Historique de l'implantation de la téléphonie mobile dans la région du Moyen-Sepik}

C'est dans les premières années du XXI ${ }^{\mathrm{e}}$ siècle que les téléphones mobiles ont commencé à être utilisés dans la capitale provinciale de Wewak alors qu'existaient depuis longtemps déjà des téléphones fixes reliés aux autres villes du pays et de l'étranger. En 2005, lors du tournage du documentaire sur l'expédition de la Korrigane (Fargier, 2005), la production de Dokumenta avait confié un téléphone Iridium au réalisateur avec lequel il pouvait entrer en contact avec elle à Paris. Les villageois d'Abusatngei ne furent pas trop surpris de voir utiliser cet appareil car ils savaient pour la plupart que les conversations codées pouvaient être transmises par l'intermédiaire d'un satellite. C'était d'ailleurs un jeu fréquent pour les enfants du village durant les soirées étoilées de rechercher dans le ciel les petits points brillants (les satellites) qui se déplacent lentement ${ }^{7}$.

En 2007, la ville de Wewak et ses environs dans la province du Sepik de l'Est furent rapidement desservis par des réseaux de téléphonie mobile Bmobile $^{8}$ et Digicel$^{9}$ (Mitchel, 2008; Watson, 2011 : 46-52 ; Lipset, 2013 et 2017 ; Telban et Vavrova, 2014 : 224-226). David Lipset (2017 : 201-206) en déduit la création d'une nouvelle sorte de "sphère public " qui implique tous les utilisateurs de téléphones mobiles. Il devint alors possible d'utiliser internet dans de petites échoppes créées par de jeunes locaux. C'est ainsi que des hommes du village de Yentchen eurent l'idée de proposer sur internet la vente des sculptures de leur vieille maison cérémonielle délabrée. Le succès de ces

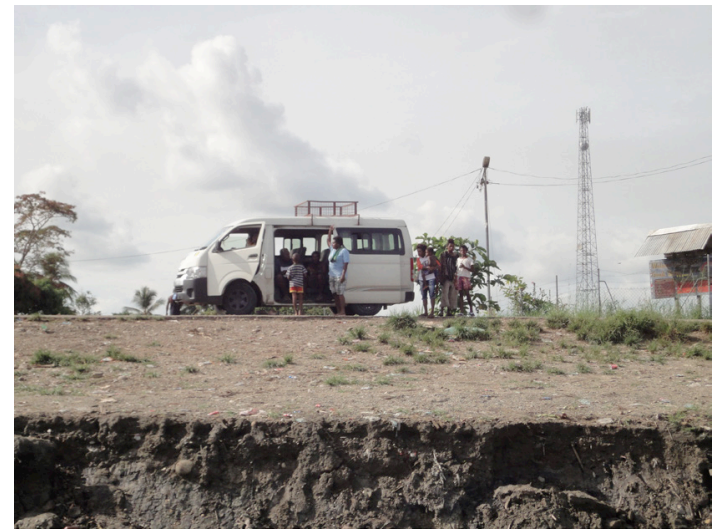

Рното 2. - Antenne-relais du village de Pagwi au bord du fleuve Sepik (C) C. Coiffier, 2010)

nouveaux moyens individuels de communication fut immédiat ${ }^{10}$ comme dans les autres états du Pacifique et les ventes de téléphones mobiles ont augmentée d'année en année (Suwamaru, 2015).

Quelques années plus tard, une antenne-relais fut implantée en 2009 dans le village de Pagwi, au débouché de la route arrivant de Maprik, puis une autre l'année suivante dans le village de Timbunke en aval. Les habitants des pentes nord-ouest des collines de Chambri et Aïbom purent alors capter des messages grâce à ces antennes-relais. C'est ainsi que pour la première fois, en 2010, j'ai pu contacter ma famille en France depuis les berges du Sepik à Ambunti. À cette époque, les habitants de la région du moyen Sepik utilisaient des cartes d'unités téléphoniques Bmobil ou Digicel qui se vendaient dans les petites épiceries locales et sur les marchés situés sur la route Pagwi-Maprik-Wewak.

La réception des communications était assez mauvaise au village de Palimbeï. Selon un de mes informateurs, il fallait parfois grimper en haut d'un arbre gupma (Neonauclea sp.) pour pouvoir capter le réseau et rentrer en contact avec un correspondant (Telban et Vavrova, 2014 : fig. 1). Constatons que le choix de ce type d'arbre n'est pas anodin lorsque l'on sait qu'il est utilisé fréquemment pour divers rituels comme celui de la nouvelle année ndemaï, annoncée par l'apparition à l'horizon de la constellation des Pléiades. Les feuilles de cet arbre sont également utilisées pour toutes sortes d'opérations magiques (Coiffier, 1994 : 621 et 926-927).

La compagnie Digicel a alors entrepris une efficace et agressive campagne publicitaire avec distribution en ville et dans les villages de parapluies et de T-shirts décorés avec son logo pour se faire

7. Mais en fait c'est un satellite géostationnaire avec des antennes relais à terre qui est utilisé pour la téléphonie mobile.

8. Société connue comme GSM system by Telikom subsidiary Pacific Mobile Communication Company Ltd en 2003, avant de devenir en 2009, la société Telikom Papua New Guinea dirigée par l'un des fils du Premier ministre Michael Somare.

9. L’opérateur Digicel Papua New Guinea (Digicel group Ltd.) a commencé son implantation locale en juillet 2007. Cette société irlandaise a débuté ses activités avec succès à Kingston en Jamaïque avant de les développer dans d'autres îles des Caraïbes et de l'océan Pacifique. Il est permis de se demander si la diffusion du Reggae a favorisé sa rapide expansion.

10. Je me trouvais au sud de l'île de Santo (au Vanuatu) en 2008 lorsque l'opérateur Digicel a mis en vente à Luganville ses premiers téléphones portables. Il y avait de longues files d'attente devant les magasins et plusieurs milliers d'appareils furent vendus en quelques jours. 


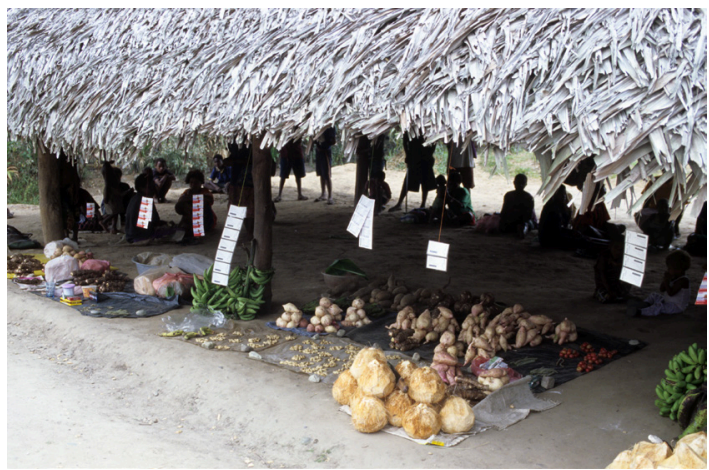

Рното 3. - Cartes Bmobil et Digicel en vente sur un marché sur la route Maprik-Wewak (C C. Coiffier, 2010)

connaitre du plus grand nombre. En mai 2011 la Société Digicel annonça qu'un nouveau réseau de téléphonie mobile utilisant la technologie 3G à haut débit avait été inauguré en Papouasie Nouvelle-Guinée (Decloitre, 2011: 3). Au début de l'année 2013, une nouvelle antenne-relais Digicel fut installée au milieu du village de Yentchen, ce qui permit aux habitants de la région de se connecter sur internet et d'accéder plus aisément au réseau. Cette antenne-relais se trouve construite sur un terrain qui appartenait autrefois au clan Tschameangwaat ${ }^{11}$ laissé à l'abandon depuis le décès de ses derniers représentants locaux. L'opérateur Digicel a donc loué ce terrain aux actuels propriétaires. Actuellement, le réseau Digicel fonctionne plus ou moins bien selon l'endroit où l'on se trouve mais il est fréquent de voir des villageois dans leur pirogue circulant à vive allure sur le fleuve en communication avec un correspondant. La connexion au réseau n'est pas toujours très bonne dans les villages Sawos situés dans la brousse (comme Nangosap, Gaïkorobi ou Marap) car ils sont trop éloignés des antennes-relais situées au bord du fleuve, si bien qu'il existe une demande grandissante auprès des autorités locales et de l'opérateur Digicel pour la construction de nouvelles installations.

Des employés de la société Digicel remontent maintenant le fleuve Sepik plusieurs fois par an et s'arrêtent quelques jours dans chacun des villages pour y proposer à la vente de nouveaux modèles de téléphones mobiles et des cartes d'unités téléphoniques. Outre le modèle basique per-

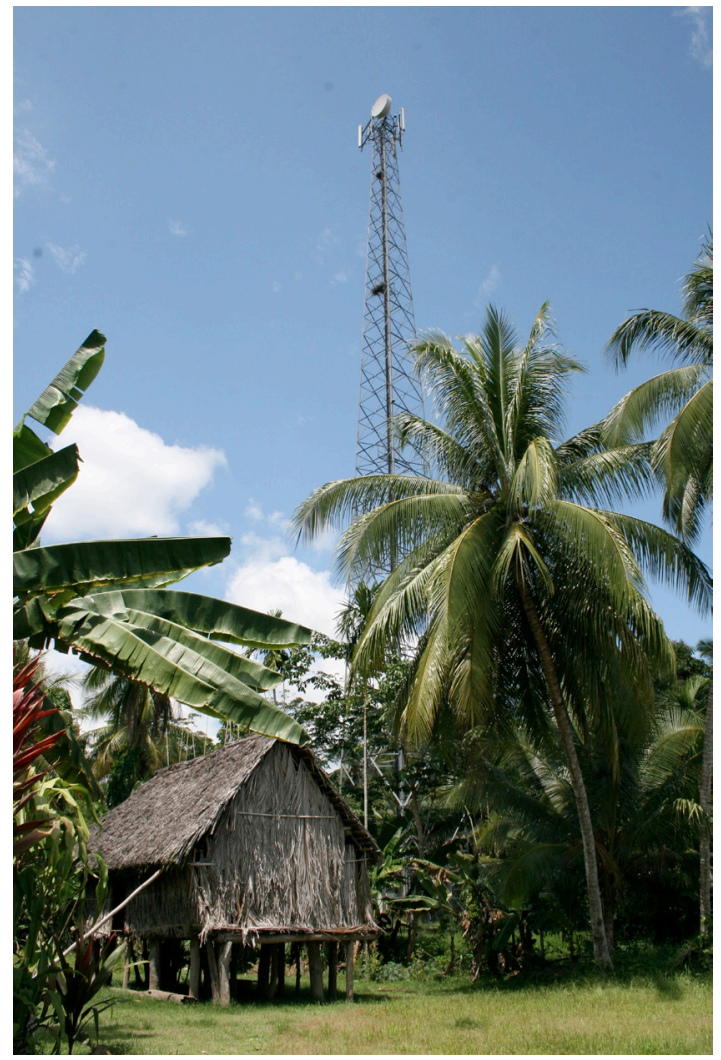

Рното 4. - Antenne-relais du village de Yentchen (C) C. Coiffier, 2015)

mettant simplement de converser, d'envoyer et de recevoir des SMs, ils présentent également des smartphones permettant de téléphoner mais aussi de prendre des photos, d'enregistrer des vidéos et d'accéder au réseau internet. C'est ainsi que, le jeudi 6 août 2015, deux employés de la compagnie Digicel sont arrivés à Abusatngei accompagné d'un policier armé d'un fusil pour les protéger des attaques des rascol ${ }^{12}$ locaux. Ils se sont installés fort opportunément dans une maison située à côté d'une place où les villageois ont l'habitude de jouer au Bingo ${ }^{13}$ avec de l'argent. C'est ainsi que les gagnants chanceux purent investir rapidement leurs gains dans du nouveau matériel téléphonique. Le vendredi, un téléphone mobile fut proposé comme lot gagnant pour la séance journalière finale de Bingo en guise de promotion de la marque.

11. Le clan Tschameangwaat à pour origine une terre située en aval du village de Tambanum. Les membres de ce clan, répartis actuellement dans différents villages, revendiquent la paternité de l'invention des pratiques de scarifications lors des initiations des jeunes hommes. Est-ce un hasard si le mot Tschameangwaat peut se traduire ainsi : « unifier ou relier les êtres qui ont été séparés " ?

12. Le terme rascol désigne en pidgin-english les délinquants, les brigands qui agressent les voitures sur les routes ou les pirogues sur le fleuve.

13. Le temps accordé à ce jeu très populaire par les habitants du hameau d'Abusatngei a beaucoup augmenté depuis vingt ans. Le jeu de Bingo accapare une partie de leur temps alors que les activités de jardinage ou de pêche sont plus ou moins délaissées. Cela semble montrer que certains habitants disposent maintenant de suffisamment d'argent pour s'acheter de la nourriture dans les magasins. Les membres de leur famille travaillant dans les centres urbains leur apportent assez régulièrement une aide financière. Les plus anciens qui ne dédaignent pas de participer aux séances de Bingo sont cependant les premiers à critiquer le temps perdus sur les activités traditionnelles (Lin, 2015). 


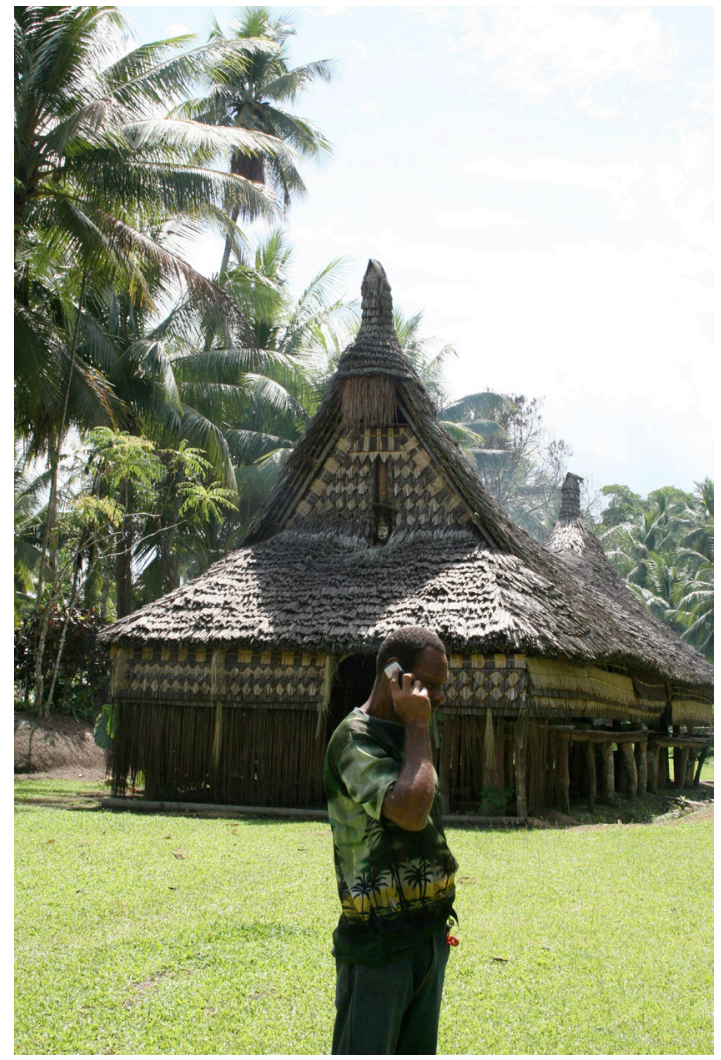

Рното 5. - Un homme du village de Kanganaman (voisin de Yentchen) téléphone devant la maison des hommes Mundjimbit (C C. Coiffier, 2015)

\section{Accès et utilisation des téléphones dans la région}

Les prix des téléphones mobiles sont relativement abordables pour les habitants des villages. $\mathrm{Si}$ le prix d'un smartphone de marque chinoise est d'environ 200 kina (70 euros environ), celui d'un téléphone mobile simple de la marque Nokia est proposé à 25 kina, (soit 9 euros environ) ce qui représente localement le prix de quelques poulets. Cependant les frais d'achat et d'utilisation d'un téléphone mobile demeurent assez onéreux pour les villageois qui ne reçoivent pas d'aide de membres de leur famille travaillant en ville. Il n'y a pas beaucoup d'opportunités de gagner de l'argent dans les villages, si bien que c'est souvent le produit de la vente aux touristes des objets fabriqués en bois qui permet à un homme bon sculpteur d'acquérir le téléphone convoité. De leur côté, les femmes se procurent l'argent nécessaire à cet achat en vendant des filets bilum aux touristes, et le produit de leur pêche ou de leur récolte de noix d'arec et de tabac sur les marchés. Ainsi, chaque genre a sa propre dynamique économique pour se donner les moyens d'acquérir un téléphone mobile, objet très personnel.

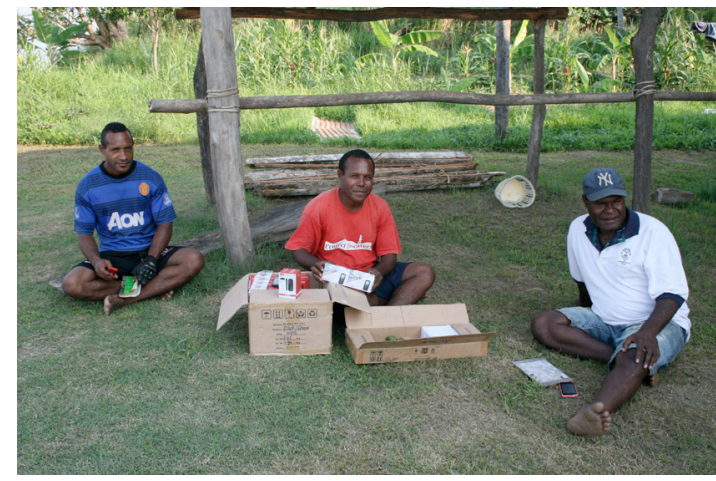

Рното 6. - Deux représentants de l'opérateur Digicel et un client installés près de la place de jeu de Bingo au village d'Abusatngeï (C) C. Coiffier, 2015)

Quelques personnes possèdent un petit ordinateur dans le village d'Abusatngei, le premier homme à posséder un tel appareil au village de Palimbei fut Teddy Balangu, un artiste reconnu qui fut invité en atelier par le musée d'ethnologie de Vancouver et par une galerie de Victoria (Canada). Lors de notre dernier séjour, en 2015, un instituteur de l'école possédait également un petit ordinateur. L'utilisation de l'ordinateur ou du smartphone nécessite de maîtriser un tant soit peu la langue anglaise pour pouvoir utiliser au mieux ces appareils ; or c'est le cas de la majorité des villageois de moins de cinquante ans qui parlent et écrivent plus ou moins cette langue actuellement.

Les ordinateurs comme les téléphones mobiles ont besoin d'énergie pour fonctionner et il n'existe pas encore de réseau de distribution d'électricité dans la région. Ce sont donc les groupes électrogènes fonctionnant avec du pétrole qui permettent de recharger les batteries des téléphones portables. Depuis une vingtaine d'années, il existe normalement dans chaque village de la région au moins un groupe électrogène, mais le prix de cet équipement reste assez élevé (de 350 kina jusqu’à 1200 kina selon la puissance). Il faut ajouter à cela le coût de l'achat et du transport du pétrole dont les prix ne cessent d'augmenter avec les années. C'est pourquoi on constate que les possesseurs d'ordinateur et de smartphone dans les villages sont en général des entrepreneurs locaux possédant déjà une pirogue à moteur leur permettant de gagner de l'argent en effectuant des transports de marchandises et de personnes. Les écoles possèdent ordinairement un groupe électrogène, ce qui permet aux instituteurs de faire fonctionner leur ordinateur et de recharger la batterie de leur téléphone mobile. Les membres de leurs familles et les parents d'élèves utilisent parfois également cet équipement.

Depuis quelques années de petits panneaux solaires sont apparus sur le marché et leurs prix les mettent à la portée de la bourse des villageois, ce qui donne l'opportunité à ces derniers d'en acheter dans les commerces chinois de Wewak pour 


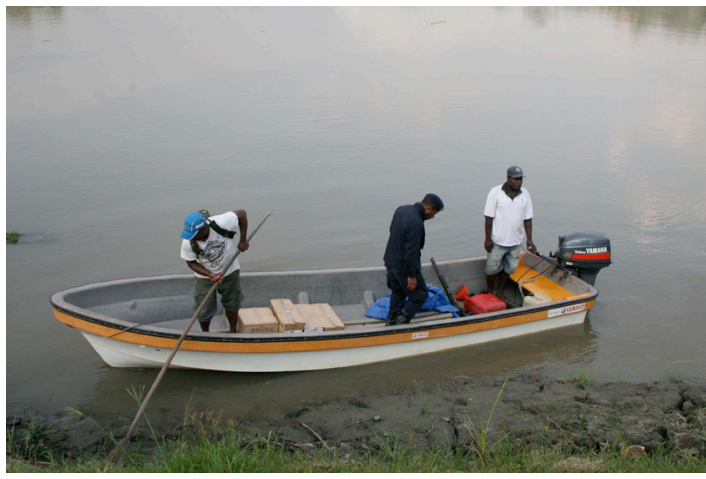

Рното 7. - Les représentants de l'opérateur Digicel accompagnés par un policier quittent le village d'Abusatngeï dans leur canot (C C. Coiffier, 2015)

des sommes allant de 120 kina (pour une taille de $20 \mathrm{~cm} \times 20 \mathrm{~cm}$ ) à 1400 kina (pour un modèle de $70 \mathrm{~cm} \times 50 \mathrm{~cm}$ ). Il n'est pas rare ainsi de voir des panneaux solaires dressés à côté des maisons au milieu des poulets et des cochons.

La majorité des téléphones mobiles fonctionnent avec des cartes d'unités téléphoniques qui permettent de les recharger. Il existe des cartes à différents prix : 3, 5, 10, 20, 50 kina. Il est également possible de transférer une certaine somme de crédit d'un compte téléphonique à un autre, c'est-àdire que le possesseur d'une carte de 20 kina peut par exemple transmettre un crédit de 5 kina à un ami. Les virements financiers à partir d'un compte en banque à Wewak peuvent passer maintenant par l'intermédiaire du réseau de téléphonie mobile.

\section{Impact de l'implantation de cette nouvelle technologie et de l'accès au web}

Les changements occasionnés par l'implantation du téléphone mobile et de l'accès au web dans une société basée sur une économie de subsistance permettent d'affirmer que les avantages sont incontestables. Dans ma famille adoptive, à Abusatngei, les parents et cinq des sept enfants disposent maintenant chacun d'un téléphone mobile. Le père Benny Kusodimi a reçu son premier téléphone mobile en 2010 comme cadeau d'un de ses fils résidant à Madang, ce qui lui a permis de communiquer régulièrement avec ces derniers et avec d'autres membres de sa famille dispersés à travers le pays. C'est ainsi qu'il fut très fier de me transmettre son numéro et que nous avons pu communiquer plus aisément entre la France et la Nouvelle-Guinée alors que les échanges de lettres prenaient auparavant plus d'un mois entre Paris et Palimbeï. Benny a développé rapidement une addiction à cette nouvelle technologie. Au début de l'année 2015,

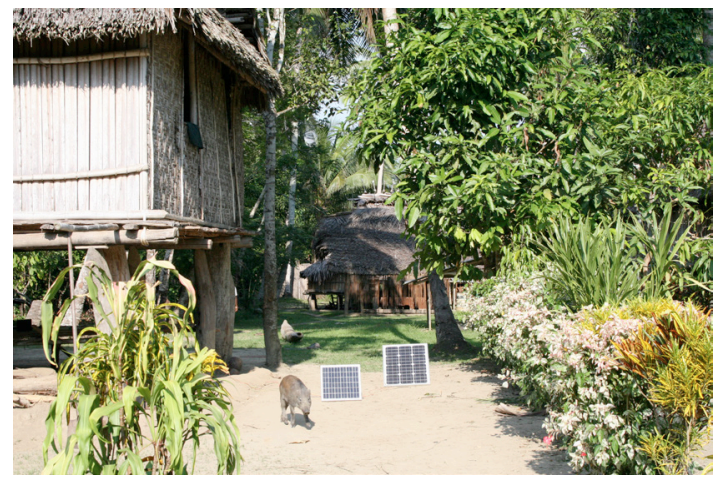

Рното 8. - Deux batteries solaires installées près d'une maison du village sawos de Nangosap (ㄷ C. Coiffier, 2015)

il m'a transmis pour la première fois des emails sur ma messagerie électronique du Muséum. Lorsque je lui ai demandé s'il avait utilisé un ordinateur à Wewak, il m'a répondu qu'il les avait envoyés depuis son nouveau smartphone. Cette réponse demeure mémorable car elle marque un grand changement sur le regard que je portais depuis une quarantaine d'années sur la société iatmul. En effet, à cette même époque, à Paris, je n'utilisais pas encore personnellement de smartphone permettant d'envoyer et de recevoir des e-mails.

Les smartphones sont pourvus de bien d'autres applications à l'usage du quotidien; ils remplacent les montres, permettent de calculer et servent d'alarme, ils peuvent également être utilisés comme un miroir pour rectifier son maquillage ou l'ordre des plumes de sa coiffure lors des fêtes. En cas de blessure grave ou d'un accouchement difficile, le téléphone permet de recevoir des conseils d'un infirmier ou d'un médecin de l'hôpital de Timbunke ou de Wewak. La téléphonie mobile modifie ainsi totalement les relations entre les villageois et les services de santé comme l'a très bien montré Henry Yamo (2013) pour la province de Western Highlands. Les réservations pour obtenir une place dans une pirogue se rendant au marché de Pagwi ou dans un PMV circulant entre Pagwi, Maprik et Wewak se font maintenant par téléphone. L'arrivée d'une pirogue dans un village est annoncée par un appel téléphonique du conducteur ${ }^{14}$. Le téléphone mobile permet le développement d'échanges ayant lieu les jours de marché et facilite la création de petites entreprises locales dans divers villages (Suwamaru, 2015 : 7). Les transferts électroniques accélèrent notamment la circulation de l'argent destiné aux rituels de mariage ou de funérailles au sein des familles comme l'ont très bien montré Singh and Yaso (2011) pour la province de Morobe. La transmission d'un message qui, il n'y a pas si longtemps se faisait par divers intermédiaires (message tambouriné, messagers, radio) peut se faire main-

14. Cependant les villageois ont depuis longtemps appris à reconnaître les bruits des différents moteurs comme dans les campagnes françaises les paysans savaient différencier de loin les différents bruits de moteurs de tracteurs Ferguson, Ford ou Mac Cormick (information personnelle transmise par Thierry Morant en 2015). 


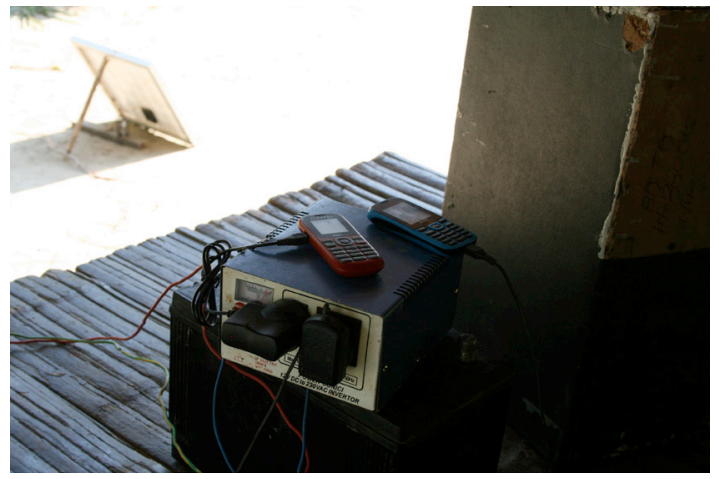

Рното 9. - Branchements de téléphones mobiles sur des batteries solaires à Nangosap (C) C. Coiffier, 2015)

tenant par le biais d'internet ou d'un message téléphoné. Outre les publicitaires et les politiciens, les nombreuses communautés religieuses chrétiennes implantées dans le pays tirent profit de ce nouveau moyen de communication pour diffuser leur enseignement et pour recruter des adeptes. Les jeunes ont ainsi la possibilité d'avoir accès à des sites islamistes intégristes et il est prématuré actuellement d'en mesurer les conséquences.

L'épouse de Benny s'énerve souvent contre son mari car elle lui reproche de passer trop de temps à jouer sur son téléphone au lieu d'aller travailler au jardin ou de lui creuser une nouvelle pirogue pour aller pêcher. En effet, j'ai pu constater lors de ma dernière visite au mois d'août 2015 que Benny utilisait fréquemment son dernier modèle de smartphone $^{15}$. Il a réussi à trouver sur la plateforme Youtube des archives cinématographiques provenant de films ethnographiques réalisés dans les années 1970 par des chercheurs allemands et suisses dans le village de Palimbeï. C'est avec émotion que je le vis présenter à ses petits enfants son père Raphaël Tambemali ${ }^{16}$, en train de fabriquer un instrument de musique local à partir d'une palme de sagoutier. La jeune génération peut ainsi visionner les activités disparues de ses ancêtres. Le soir toute la famille se réunit après le dîner pour regarder ces vieux documentaires qui rappellent leur jeunesse aux plus anciens. internet donne ainsi aujourd'hui la possibilité aux femmes et aux non initiés de visionner en secret des cérémonies qui leurs étaient autrefois totalement interdites. Si certains hommes font une distinction entre la vision directe et la vision sur un écran, il n'est pas exclu que ces changements participent à mettre un terme dans l'avenir aux initiations et à certains interdits dévolus aux femmes et aux non initiés. Comme cela se produisit dans

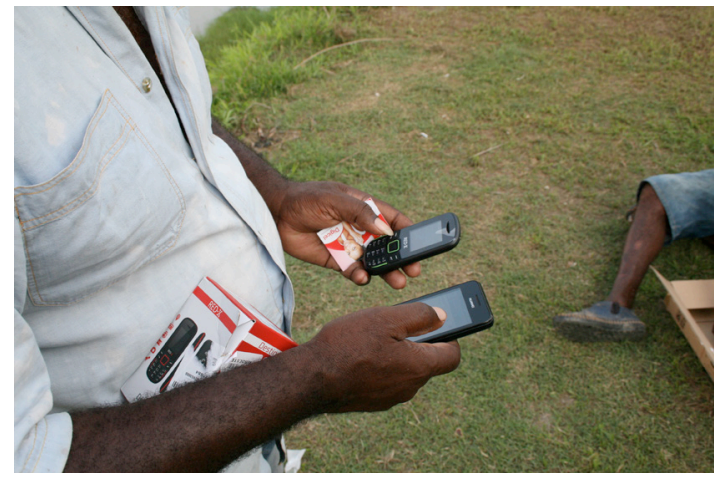

Рното 10. - Un choix difficile ! Lequel des deux, mobile phone ou smartphone? Au village d'Abusatngeï (C) C. Coiffier, 2015)

les années 1960, lorsque de nouveaux convertis à la religion chrétienne présentèrent aux femmes les flûtes "sacrées", ce qui mit fin subitement à la vie cérémonielle antérieure. De même, les sculpteurs du village ont maintenant la possibilité d'accéder, aux résultats des ventes aux enchères publiques à Sydney ou à New-York. Ils découvrent alors les marges importantes pratiquées par les marchands entre les prix d'achat et les prix de vente d'objets anciens ou récents provenant de leur région. Ceci a pour conséquence de faire augmenter les prix de l'artisanat local, tout particulièrement pour les objets les plus anciens. Les villageois peuvent également avoir accès plus aisément aux écrits des ethnologues qui ont travaillé avec eux et ils peuvent ainsi en apprécier ou en critiquer la pertinence.

Grâce aux ordinateurs et aux téléphones mobiles, les instituteurs de l'école voisine sont maintenant en contact tant avec les parents d'élèves qu'avec leurs hiérarchie en ville; ils ont aussi accès aux bibliothèques et autres lieux de savoir ${ }^{17}$. Mais l'accès de certains élèves aux téléphones mobiles commence à poser des problèmes comme dans d'autres pays mélanésiens. C'est ainsi que le ministre fidjien de l'éducation, Filipe Bole a déclaré :

«Notre politique d'interdiction de l'usage des téléphones mobiles pendant les heures de cours est toujours en vigueur. Elle a pour objet d'apprendre à nos élèves que l'école est un lieu d'apprentissage et non pas un lieu pour passer son temps soit à parler au téléphone, soit à écouter de la musique. Et il est reconnu que les téléphones mobiles sont une source de distraction pendant les heures de cours, ce qui a pour effet d'irriter les enseignants. » (Decloitre, 2011 : 4)

Les habitants du Sepik apprécient beaucoup les photographies ${ }^{18}$ et chaque famille possède son pe-

15. Ce téléphone est très utile pour Benny dans le cadre de ses nouvelles fonctions d'assesseur juge contumier (Village Court magistrate) pour le village de Palimbeï.

16. Raphaël Tambemali fut un de mes informateurs dans les années 1980.

17. Un plan GPE (Global Partnership for Education) pour une éducation de base universelle a été mis en place en Papouasie Nouvelle-Guinée de 2010 à 2015 avec l'aide de la banque mondiale.

18. Le terme kaïk désigne en langue Iatmul à la fois le double d'une personne et une photographie (Coiffier, $2000: 268$ ). Ainsi les images des écrans de télévision et de smartphone sont maintenant considérées comme des kaïk. 
tit album avec des images jaunies et souvent rongées par les moisissures ${ }^{19}$. Avant l'apparition des téléphones permettant des prises de vue et des vidéos, peu de villageois avaient investi dans l'achat d'un appareil photographique dans la mesure où il était difficile et coûteux de faire développer les pellicules à Wewak (Coiffier, 2000). Ils leurs fallait donc attendre souvent assez longtemps pour voir les photos tirées sur papier. Les nouveaux modèles de téléphones mobiles simples combinent néanmoins des fonctionnalités comme la réception de radios $\mathrm{FM}$, la lecture de fichiers MP3, ou encore la prise de photos et l'enregistrement de vidéos (Decloitre, $2011: 4$ ). Ils permettent d'obtenir des images visibles immédiatement sur écran. Lors des fêtes singsing, il est maintenant commun de voir des villageois prendre des photos du spectacle et surtout de leurs amis ou membres de leur famille en costume local. Comme partout dans le monde les jeunes dans les villages apprécient particulièrement de se prendre en photo et nombreux utilisent l'application Snapchat permettant de transformer les images avec divers montages. C'est ainsi qu'ils s'amusent à mettre la tête d'une personne sur le corps d'une autre, parfois ce peut être une tête d'un animal qui évoque un personnage de la mythologie locale comme Mandawa, la femme à tête d'oiseau, Kwolibank, le chien chauve-souris ou le cochon Gusui à tête de rat, ancêtre du clan Wanguande (Coiffier, 1994: 325-328 et 621). L'aide entre amis pour accéder à de nouvelles applications fait partie des nouveaux rapports de convivialité. Celui qui maîtrise le mieux ces fonctionnalités acquiert un grand prestige auprès de ses camarades. Si autrefois un homme essayait de connaître les différents noms familiaux d'une femme convoitée, pour pratiquer diverses magies, c'est maintenant son numéro de téléphone qu'il essaye d'obtenir pour un usage similaire.

Comme partout ailleurs dans le monde, l'utilisation du téléphone mobile et l'accès au web ne présentent pas que des conséquences positives sur les rapports humains. C'est ainsi quau mois de septembre 2007, alors que nous quittions de nuit la ville de Wewak en direction de Pagwi, assis dans un $\mathrm{PMV}^{20}$ en compagnie d'une quinzaine de personnes au milieu de filets remplis de vêtements, de sacs de riz, de cartons de boîtes de conserve et de matelas, une passagère vint perturber notre petit groupe ; celle-ci se mit à utiliser son téléphone en conversant à voix haute avec une amie durant presque une demi-heure. Plusieurs passagers montrèrent leur mécontentement en faisant des commentaires et des remarques sur ses propos sans que cela ne mette fin à sa conversation qui ne s'arrêta que lorsque son téléphone n'arriva plus à capter le réseau. Il est probable que les passagers se seraient dispensés de leurs commentaires si le locuteur avait été un homme.

Larrivée du téléphone mobile dans la région a également permis aux rascol de développer leurs agissements. C'est ainsi que dans les années 20122013 un groupe de délinquants écumait la région du bas Sepik sur des bateaux pirates et rançonnait touristes et villageois. Ils pouvaient aisément communiquer entre eux grâce à cette nouvelle technologie, mais la police a rapidement mis fin à leurs activités délictueuses. Les réseaux téléphoniques permettent la diffusion dans les villages de brousse de rumeurs plus ou moins fondées, comme une histoire de médecins véreux qui feraient assassiner des hommes et des femmes à Wewak par des rascol pour leurs prélever divers organes qui seraient ensuite vendus à l'étranger. Ces rumeurs sont d'autant plus effrayantes que nombre de villageois vivent dans un milieu où la sorcellerie et les meurtres rituels n'ont jamais totalement cessé. Les possibilités de manipulations de messages téléphoniques par des personnes mal intentionnées sont de plus en plus nombreuses et permettent l'émergence de nouvelles pratiques de sorcellerie. Lamont Lindstrom (2011 : 263) fait le même constat au Vanuatu.

\section{Effets du téléphone portable sur la sexualité locale}

David Lipset (2013) perçoit dans l'utilisation des réseaux sociaux un renforcement de l'égocentrisme au dépend de l'ancienne sociabilité et il constate une dissolution de la morale introduite depuis un siècle par les divers évangélisateurs. Les mariages et les relations extraconjugales ont bien changé depuis un siècle, lorsque de nombreux hommes du Sepik ont été recrutés par les administrations coloniales successives pour travailler dans les grandes plantations de cocotiers du nord de la Nouvelle-Guinée et de Nouvelle-Bretagne. Les mariages arrangés (ou forcés) par les familles sont beaucoup moins fréquents quautrefois. De nos jours, les mariages sont plus souvent basés sur des choix personnels. La téléphonie sans fil vient bouleverser les règles établies car elle élargie la sphère des relations possibles et facilite les contacts. Ce phénomène a été observé dans les autres états mélanésiens comme le Vanuatu (Servy, 2013 ; Taylor, 2016) et les îles Salomon (Hobbis, 2017). C'est ainsi qu'Alice Servy (2013 : 141) évoque l'étonnante réponse d'une de ses informatrices à sa question :

19. Ces photographies avaient été souvent réalisées par des touristes ou des missionnaires de passage qui les avaient ensuite fait parvenir aux intéressés.

20. Les pmv (Private Motor Vehicule) sont des camions bâchés avec des bancs de bois placés sur chacun des côtés longitudinaux de la benne. Ils appartiennent à des entrepreneurs locaux qui transportent, avec une régularité de deux à trois voyages aller-retour par semaine entre Wewak et Pagwi, du fret (barils de pétrole et sacs de riz vers le fleuve, sacs de café et paniers de poissons séchés vers la ville) et une vingtaine de passagers accompagnés de leurs bagages. 
"As-tu un petit copain?"

«Non je n’ai pas de téléphone!»

Le téléphone portable est donc devenu pour les jeunes mélanésiens, comme ailleurs, plus qu'un outil de communication. Le téléphone mobile, objets très personnels contenant parfois des informations intimes, doit être toujours porté sur soi. C'est ainsi que les villageois du Sepik le transporte dans leur petit filet ${ }^{21}$ bilum, avec le tabac, les noix d'arec, les fruits de bétel et la boîte de chaux, éléments traditionnellement associés à la communication avec les êtres et les esprits. Ces divers éléments destinés à la fabrication d'une chique de bétel sont souvent utilisés comme petits cadeaux prémices à une séduction amoureuse.

Selon Macdonald et Kirami (2017: 112-116) l'utilisation des téléphones mobiles aurait un impact particulier sur le statut des femmes. Il est vrai qu'ils leurs apportent, en plus de leur scolarisation, une nouvelle ouverture sur le monde avec la possibilité de contacter un médecin lors d'un accouchement difficile et d'entrer en relation avec des associations féminines, par exemples. Ce qui est le cas de l'épouse de Benny qui fait partie d'une association de prévention des maladies sexuellement transmissibles.

Lors de notre dernier séjour à Palimbei en 2015 nous avons pu constater que Benny Kusodimi utilise l'application Facebook et qu'il a des centaines d'"amis" virtuels dans le monde entier. Son épouse Martha nous fit même remarquer qu'il a une amie au Brésil avec laquelle il converse assez souvent en langue anglaise. Hommes et femmes cherchent de nouveaux partenaires sur des sites de rencontres récemment créés en Papouasie Nouvelle-Guinée ; il en existe maintenant plusieurs qui sont très populaires. Les SMS sont très pratiques pour donner des rendez-vous galants dans des lieux secrets de la brousse. Mais lorsqu'une femme découvre que son mari à une relation téléphonique ou par internet avec une autre femme, elle confisque son téléphone et le jette dans le fleuve Sepik. Selon les dires des villageois d'Abusatngei, des dizaines de téléphones mobiles se trouveraient maintenant au fond de l'eau au milieu des esprits des eaux wagan ${ }^{22}$ (Coiffier, 1994 : 1295) ce qui est sensé effrayer les maris volages et les ramener à la raison dans la mesure où ces derniers croient encore à la puissance de ces esprits. Une histoire m'a été racontée comme quoi une femme de Rabaul s'est trompée de correspondant en téléphonant à un homme de Palimbei. Elle proposa cependant à celui-ci de correspondre avec lui et éventuellement de le rencontrer à Wewak. Le mari de cette femme ayant entendu la conversation devint furieux et commença à la frapper. Il lui arracha son téléphone pour faire des menaces au correspondant. Ainsi l'utilisation des téléphones mobiles semble jouer un rôle amplificateur dans les conflits conjugaux de nombreux villages de Nouvelle-Guinée comme dans les autres états mélanésiens (Servy, 2013 : 141 ; Hobbis, 2017). Il est possible qu'il exacerbe plus qu'auparavant les sentiments de jalousie dans la mesure où les concurrents sont plus difficilement identifiables. Au début de l'année 2010, la presse électronique (Decloitre, 2010 : 1-2) annonçait :

«Papouasie Nouvelle-Guinée : Deux morts par SMs. »

Dans la région de Tari (Province de Hela), suite à la réception par une femme de la tribu des Pipi d'un SMS à connotation érotique, voir pornographique, de la part d'un jeune homme du clan Tapo, ses frères organisèrent une expédition punitive pour laver l'honneur de leur clan. Il s'en suivi un conflit entre deux villages qui se solda par la mort de deux hommes, plusieurs blessés, une quinzaine de maisons incendiées, des magasins pillées et des champs de cultures vivrières détruits.

Les adolescents, quant à eux, profitent de certains sites internet pour visionner des images pornographiques, ce qui inquiète beaucoup leurs parents et les instituteurs. Certains expérimentent ainsi de nouvelles pratiques sexuelles perçues sur ces images ou sur ces films. D'autres s'échangent des recettes de fabrication d'alcool à base de divers fruits locaux ou de mélanges de divers stupéfiants. Les responsables des communautés religieuses locales s'inquiètent et essayent d'interdire ou de contenir ces nouvelles pratiques, pas toujours avec le succès escompté. Des hommes des villages du Sepik utilisent internet pour se faire livrer (en ville) des médicaments, comme le Viagra, pour conforter leur vigueur sexuelle. Le réseau internet permet cependant aux associations de transmettre des informations concernant la protection contre les maladies sexuellement transmissibles et particulièrement contre le virus VIH.

\section{Les nouvelles technologies et les effets de l'implantation des sociétés multinationales dans la région}

Si le développement de la téléphonie mobile dans la région du fleuve Sepik permet aux popu-

21. Les femmes fabriquent de petits filets bilum adaptés tout spécialement au transport des téléphones mobiles. Cette pratique existe dans d'autres régions de Papouasie Nouvelle-Guinée, comme dans d'autres pays, avec des matériaux très divers. J'ai constitué une petite collection de ces filets qui se trouve conservée maintenant au musée de l'Homme. Une vitrine du parcours permanent est dévolue à la présentation de quelques spécimens.

22. Chaque clan possède ses propres paires d'esprits des eaux, wagan, dont la présence est visible dans les tourbillons du fleuve. Ils sont sensés être très puissants puisqu’ils ont la réputation de pouvoir faire chavirer une pirogue. 
lations locales d'avoir un nouveau regard sur le monde, il faut constater qu'il a facilité l'implantation des compagnies forestières. C'est en effet après la construction des antennes relais à Pagwi, Timbunke et Yentchen que ces compagnies se sont installées dans la région et ont commencé leurs activités destructives pour l'environnement. Ces sociétés ne créent guère d'emplois locaux car elles déménagent une fois les abattages terminés.

C'est une compagnie malaisienne qui a été chargée de la construction d'une route à partir du village de Yentchen en échange de concessions de parcelles forestières à exploiter. Une vaste surface de forêt à été abattue à cent mètres en aval de ce village non loin de l'antenne-relais Digicel. Deux ponts de bois et un débarcadère pour le chargement des grumes sur des barges ont été construits. À cet emplacement se trouvait le $s a a^{23}$ (Coiffier, 1994: 425) de la maison des hommes Nyanglambi qui était utilisé lors des initiations. Il a été entièrement détruit et des baraquements en bois ont été construits non loin de là pour loger les employés malais. En 2015, le débroussaillage du tracé de la nouvelle route était terminé jusqu'aux villages de Nangosap et de Gaïkorobi. Cette route destinée à désenclaver la région devrait relier Yentchen directement à Wewak en passant par le village de Kubalia avec une bretelle la reliant à la route existante Pagwi-Maprik-Wewak. Elle devrait ainsi desservir les villages de Nangosap, Gaïkorobi, Marap, Yamok et Torembi. Une ligne électrique devrait être construite parallèlement à cette nouvelle route pour fournir l'électricité aux différents villages de la région. Selon certains responsables locaux les villages de Palimbei et de Malingei se trouvant de l'autre côté du fleuve pourraient même être électrifiés en plaçant un câble qui traverserait le fleuve Sepik. De nombreuses réunions ont eu lieu entre les différents komiti ${ }^{24}$ de villages, et des enquêtes ont été effectuées dans les communautés religieuses pour recueillir l'avis de tous. Les téléphones mobiles ont donc été bien pratiques pour organiser ces réunions et convoquer les participants. Mais depuis 2015, les travaux de cette nouvelle route n'ont guère progressé. Les politiciens locaux ont pourtant annoncé aux villageois que nombreux projets se grefferaient sur le programme de réalisation de cette route. La construction d'un gros bourg entre Nangosap et Gaïkorobi avec la création d'un grand supermarché Papindo, un office bancaire, un petit hôpital, un poste de police et peut-être une école. Un tel centre se situerait à mi-distance des bourgs existants de Pagwi et d'Angoram ( $c f$. carte 1). Un projet de réalisation d'une grande plantation de palmiers à huile dans la région de Gaïkorobi est également prévu, mais celui-ci ne recueille pas l'agrément de la majorité des villageois qui ont bien compris que leur environnement allait être complètement bouleversé et détruit. Les grandes compagnies font valoir que cette nouvelle plantation donnera du travail à la jeunesse locale. $\mathrm{Si}$ tous ces grands projets recueille particulièrement l'assentiment des jeunes générations, les anciens y voient de leur côté une dégradation de leur manière de vivre avec une augmentation de la délinquance et de la prostitution.

Dans l'immédiat les habitants des villages concernés constatent amèrement que les meilleurs arbres kwila et miamba (Intsia bijuga et Vitex Confossus) ont été coupés par la société forestière malaisienne et envoyés vers une scierie en aval du fleuve. Si les propriétaires de parcelles sont dédommagés pour tous les palmiers et arbustes détruits lors de l'abattage des grands arbres, ils se demandent cependant comment ils vont dorénavant pouvoir construire leurs maisons et tailler de nouvelles pirogues. La société forestière procède souvent par un grossier chantage auprès des politiciens locaux qui ont souvent des difficultés à imaginer les réels enjeux de ces grands chantiers; c'est ainsi qu'elle leurs dit pour les convaincre :

"Si vous ne nous laissez pas exploiter les arbres qui se trouvent dans vos forêts nous n'aurons pas assez d'argent pour construire la nouvelle route. »

De plus, les responsables de la compagnie attisent les dissensions entre les membres de clans différents et parfois opposés pour imposer leurs prérogatives. Depuis 2011, des représentants de l'organisation non-gouvernementale wwF (World Wide Fund for nature) viennent de temps à autre dans les villages instruire les jeunes générations au sujet des conséquences néfastes de la déforestation sur l'environnement de la région et de la planète toute entière ${ }^{25}$. Les habitants de Palimbeï et des villages voisins sont ainsi informés sur les conséquences de la prochaine exploitation de la mine de cuivre et d'or sur les bords de la rivière Frieda en amont. Ils savent maintenant grâce aux informations apportées par les téléphones mobiles et par les associations de défense de l'environnement comme le wwF que l'eau qu'ils boivent et dans laquelle ils se lavent va prochainement être polluée par les produits chimiques déversés. Ils savent que les poissons qu'ils pêchent risquent d'être bientôt impropres à la consommation, même si les analyses effectuées semblent prouver le contraire. Grâce à internet, ils sont au courant maintenant de ce qui s'est passé il y a quelques années à Porgera (Burton, 2014) et dans la région du fleuve Fly, actuellement com-

23. Le saai est un lieu dans la brousse au bord du fleuve où les nouveaux initiés peuvent pratiquer hors de la vue des femmes certaines activités secrètes comme l'apprentissage du maniement des rhombes ou l'utilisation des longues flûtes en bambou.

24. Les komiti (terme pidgin-english) sont les membres d'un conseil municipal.

25. Le site de wwF peut être consulté par les internautes locaux qui désirent obtenir plus d'informations. 


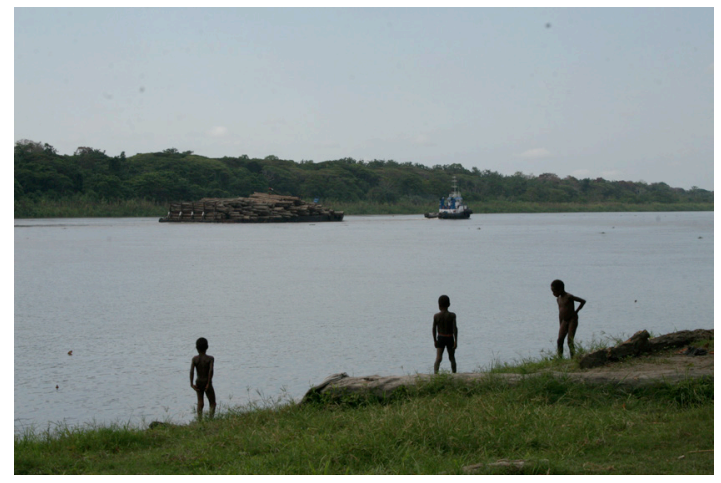

Рното 11. - Enfants du village d'Abusatngeï regardant le départ d'une barge chargée de grumes destinées à la scierie en aval (C) C. Coiffier, 2015)

plètement polluée par les déchets des mines d'Ok Tedi (Jorgensen, 2014). On assiste ainsi à une prise de conscience politique qui se développe rapidement dans diverses régions du pays (Logan, 2012). Les responsables de la compagnie minière ont annoncé qu'il y aurait deux possibilités pour exporter le minerai, soit par voie fluviale en descendant le fleuve Sepik, soit par une route à construire en amont d'Ambunti vers la côte nord de la Nouvelle-Guinée. Les habitants des villages iatmul sont opposés au premier projet car ils pensent, à juste titre, que le trafic de barges descendant le minerai vers l'estuaire du fleuve Sepik va perturber gravement la faune halieutique dont ils se nourrissent. Tous les habitants n'ont pas encore pris pleinement conscience des nombreuses perturbations collatérales qu'apportera cette nouvelle mine ; prostitution, développement de l'insécurité, développement du nombre des petits orpailleurs utilisant des produits dangereux comme le mercure avec une altération de leur environnement.

\section{Conclusion}

En quelques années l'utilisation des téléphones mobiles et d'internet est venue révolutionner, comme en de nombreux endroits de la planète, les relations humaines de la région du moyen Sepik. La Papouasie Nouvelle-Guinée est un pays jeune avec un des plus forts taux démographique au monde (2,5 millions d'habitants en 1970 et 8 millions en 2016). Selon les données de la Banque Mondiale ce pays comptait en 2011 : 140276 internautes dont 7500 abonnés haut débit, 2400000 abonnés à un téléphone fixe ou mobile. Il est significatif d'observer le décollage du nombre d'abonnés au téléphone qui passe de 100000 en 2006 à 2400000 en 2011 . Alors que le nombre d'abonnés à un téléphone fixe n'a augmenté que de 61500 à plus de 121170 durant la même période. Selon une étude plus récente (Suwamaru, 2014) $41 \%$ de la population disposerait d'un téléphone mobile. Si le développement des transports par voie terrestre ou aérienne avait déjà rapproché physiquement les communautés éloignées, le téléphone mobile permet maintenant à tout et à chacun de communiquer avec une autre personne dans les régions où sont implantés des relais. On assiste notamment selon Logan (2012), Andersen (2013) et Macdonald/Kirami (2017) à de réels changements dans la vie sociale des femmes avec la constitution d'une société civile et le développement d'une identité nationale. La téléphonie mobile joue également un rôle crucial, tant positifs que négatifs, dans le développement socioéconomique du pays comme le montrent les nombreuses et récentes recherches déjà réalisées par des Universitaires dans les diverses provinces de Papouasie Nouvelle-Guinée (Watson, 2011 et 2015; Yamo, 2013 ; Telban et Vavrova, 2014; Suwamaru, 2014, 2015 et 2016). Mais, il existe une ambivalence en ce qui concerne les conséquences du développement des technologies de l'information et de la communication. $\mathrm{Si}$ celles-ci facilitent les relations entre personnes d'un même groupe séparées entre villages et zones périurbaines, elles participent à l'augmentation de leurs déplacements et poussent les jeunes ruraux à quitter leur village pour la ville.

L'utilisation du téléphone mobile a donc permis aux habitants des villages du Sepik de sauter plusieurs étapes du développement technologique; installation d'ordinateur, achat d'appareils photos et installation d'un réseau de téléphone fixe qui aurait été beaucoup trop coûteux dans cette région. Nombreux sont ceux qui semblent accepter la déforestation et la pollution minière comme une chose inéluctable allant de paire avec le développement des nouvelles technologies dans leur région. La téléphonie mobile et internet représentent cependant un outil nouveau pour engager et organiser une riposte contre les ambitions destructrices des sociétés multinationales.

\section{BIBLIOGRAPHIE}

Aitken C. Thomas, 1990. Tapets: Drumsignals of the Sawos, in N. Lutkehaus et al., Sepik Heritage. Tradition and Change in Papua New Guinea, Durham, North Carolina, Carolina Academic Press, pp. 546-547.

Andersen Barbara, 2013. Triks, Lies and Mobile Phones: 'Phone Friend' Stories in Papua New Guinea, A Journal Culture, Theory and Critique 54 (3), pp. 318-334.

Bateson Gregory, 1958 [1936]. Naven. A survey of the problems suggested by a composite picture of the culture of a New Guinea tribe drawn from three points of view, Stanford (California), Stanford University Press (trad. française : J.-P. Latouche 
et N. Safouan, 1971. La cérémonie du naven, Paris, Éditions de Minuit).

Burton John, 20I4. Agency and the "Avatar» narrative at the Porgera gold mine, Papua New Guinea, Journal de la Société des Océanistes 138-139: Les mises en récit de la mine dans le Pacifique, pp. 37-51 (https://journals.openedition.org/jso/7118?lang=en\#toc).

CoIffier Christian, 1994. "L'écorce et la moelle du rotin ", Tshimbe kuvu, kwiya kuvu, conception iatmul de l'univers (Papouasie), thèse de Doctorat en Anthropologie sociale et Ethnologie, Paris, EHEss.

—, 1995. "Le crocodile à moteur ", les pirogues et leur motorisation chez les Iatmul du fleuve Sepik (Papouasie Nouvelle-Guinée), in A. Geistdoerfer, I. Leblic et J. Matras-Guin (éds), Les hommes et les bateaux, Usages, appropriation et représentations, Paris, CETMA, Anthropologie maritime 5, pp. 63-78, 3 fig., 5 tab.

—, 1998. Du Sagou au riz, du poisson frais au poisson en boîte en Papouasie Nouvelle-Guinée, Techniques et culture 31-32: Dynamique des pratiques alimentaires, pp. 347-362.

—, 1999. Comment nommer ces étranges objets venus d'ailleurs? Exemple des Iatmul de Papouasie Nouvelle-Guinée, in J.-L. Jamard, A. Montigny et F. Picon (dir.), Dans le sillage des techniques, Hommage à Robert Cresswell, Paris, L'Harmattan, pp. 81-105.

—, 2000. Safari photo et chasse aux têtes en Nouvelle-Guinée, Journal des Anthropologues 80-81 : Questions d'optiques. Aperçus sur les relations entre la photographie et les sciences sociales, pp. 259-281.

Decloitre Patrick Antoine, 2010 (27 janvier). Papouasie-Crimes-violence-télécom : Papouasie Nouvelle-Guinée: deux morts par sms, Flash d'Océanie, pp. 1-2 (http://newspad-pacific.info/).

—, 2011 (12 mai). Papouasie-télécommunications-mobile-internet 3G: Nouvelle mise en place de la 3G en Océanie, Flash d'Océanie, pp. 3-4 (http://newspad-pacific.info/).

FARGIER Jean-Paul, 2005. Les voyageurs de La Korrigane : sur la piste des Arts premiers, film documentaire, DVD, 52 min, Dokumenta/RMN.

Новвіs Geoffrey, 2017. A technographic investigation of mobile phone adoption in the Lau Lagoon, Malaita, Salomon Islands, thèse de doctorat en anthropologie sociale et ethnologie, sous la direction de Pierre Lemonnier et de Christine Jourdan, Paris, EHEss.

Jorgensen Dan, 2014. Mining narratives and multiple geographies in PNG: Ok Tedi, the emerald cave and Lost Tribes, Journal de la Société des Océanistes 138-139: Les mises en récit de la mine dans le Pacifique, pp. 23-37 (https:// journals.openedition.org/jso/7117).

Lin C.H., 201 5 , Pigs for Money, in C. Toren and S. Pauwels (eds), Living Kinship in the Pacific, New York, Oxford, Berghahn, pp. 36-59.

LindSTROM Lamont, 2011. Urbane Tannese: local Perspectives on Settlement Life in Port Vila, Journal de la Société des Océanistes 133: Trentenaire de l'Indépendance du Vanuatu, pp. 255-266 (https://journals.openedition. org/jso/6461).

Lipset David, 2013. Mobail: Moral ambivalence and the domestication of mobile telephones in peri-urban Papua New Guinea, Culture, Theory and Critique 54 (3), pp. 335-354.

—, 2017. Mobile telephones as public sphere in peri-urban Papua New Guinea, Journal de la Société des Océanistes 144-145: L'urbanisation en Mélanésie, pp. 195-208 (https://journals. openedition.org/jso/7835).

Logan Sarah, 2012. Rausim! Digital Politics in Papua New Guinea, Canberra, Australian National University, SSGM. Discussion Paper 9, pp. 1-16.

Macdonald Fraser and Jonathan Kirami, 2017. Women, mobiles phones and contemporary New Guinea Highlands warfare, The Australian Journal of Anthropology 28 (1), pp. 104-119.

Mc LuHAn Marshall, 1964. Understanding Media: The Extensions of Man, Corte Madera, Gingko Press (version française : 2000. Pour comprendre les médias. Les prolongements technologiques de l'homme, Paris, Seuil, coll. Points.

Mitchel R., 2008. Pathways to Telecommunication Reform, in B. Gomez (ed.), Papua New Guinea Yearbook 2008, Port Moresby, The National and Cassowary Book, pp. 134-137.

Servy Alice, 2013. "As-tu un petit copain ? Non je n’ai pas de téléphone. " Moralité, progrès technique et sexualité en milieu urbain au Vanuatu, Hermès 65 (1) : Le monde Pacifique dans la mondialisation, pp. 137-143.

Singh Supriya and Nadarajah Yaso, 2011. School Fees, Beer and "Meri": Gender, Cash and the Mobile in the Morobe Province of Papua New Guinea, IMTFI, Institut for Money, Technology and financial inclusion, Working Paper 2011/3, pp. 1-10.

SuWAMARu Kim Joseph, 2014 (12/09). Impact of mobile phone usage in Papua New Guinea, ANU press (http://www.ips.cap.anu.edu.au/ssgm).

-, 2015 (May). Aspects of Mobil phone usage in Papua New-Guinea: A social-economic per- 
spective, Divin Word University Research Journal 22, pp. 1-16.

-, 2016 (May). Modification of Dor theory - the case of mobile phones in rural Papua New Guinea, Divin Word University Research Journal 24, pp. 1-17.

TAYLOR John P., 2016. Drinking Money and Pulling Women: Mobile Phone Talk, Gendre and Agency in Vanuatu, A Journal of Social Anthropology and Comparative Sociology 26 (1), pp. 1-16.

Telban Borut et Daniela Vavrova, 2014. Ringing the living and the dead: Mobile phones in a Sepik Society, Taja, The Australian Journal of Anthropology 25 (2), pp. 223-238.

Watson Amanda Hazel Ann, 2011. The Mobil Phone: The new communication drum of Papua New Guinea, PhD Thesis, Unpublished doctoral dissertation, Queensland University of Technology, Australia.

Watson Amanda Hazel Ann and Lee R. DuffIELD, 2015 (27 décembre). From Garamut to mobile phone: communication change in rural Papua New Guinea, "Mobile media et communication", SAGE Journal.

Yamo Henry, 2013. Mobil Phones in rural Papua New Guinea: A transformation in health communication and delivery services in Western Highlands province, Unpublished master's dissertation, Auckland University of Technology, New Zealand.

Zemp Hugo et Christian Kaufmann, 1969. Pour une transcription automatique des "langages tambourinés » mélanésiens (Un exemple Kwoma, Nouvelle-Guinée), L'Homme 9 (2), pp. 38-88. 